\title{
POLITIQUES PUBLIQUES ET INTIMITÉ FACE À LA MALADIE GRAVE
}

Situer le rôle des proches aidants en Suisse

\author{
Yannis Papadaniel et Marc-Antoine Berthod
}

\section{Introduction : enjeux sociodémographiques et accompagnement des malades}

En raison du vieillissement de la population et de la chronicisation d'un nombre croissant de maladies - résultant notamment des innovations médicales et techniques -, la demande en soins à domicile va croissante. Les femmes ont longtemps été affectées à ces tâches d'accompagnement pour seconder les professionnels des soins. Si elles continuent de l'être, les changements sociodémographiques et les reconfigurations des modèles familiaux survenus au cours du $\mathrm{XX}^{\mathrm{e}}$ siècle ont cependant changé la donne : les femmes participent désormais très activement au marché du travail et les secteurs économiques ne sauraient se passer de cette importante main-d'œuvre. L'Organisation de coopération et de développement économique (OCDE), depuis 2011 (voir The Future of Families to 2030. A Synthesis Report), préconise de ce fait la mise en place de politiques sociales tenant compte de la réalité des proches ainsi que d'une prise en charge à domicile adaptée aux exigences actuelles.

Cette volonté est d'autant plus aiguë qu'une pénurie de professionnels qualifiés est attendue dans les années à venir. Les courbes démographiques du vieillissement de la population confirment la tendance à la « remédicalisation du foyer ». À ce titre, les proches se transforment bien souvent en intervenants de première ligne sans qu'il ne soit tenu compte des difficultés particulières auxquelles ils sont confrontés. Celles et ceux que l'on désigne sous des appellations aussi diverses que « aidants familiaux », « proches aidants » ou « aidants naturels ${ }^{1}$ »

1. Pour notre part, lorsque nous évoquons les catégories d'aidant informel ou de proche aidant, nous nous référons aux individus qui entreprennent régulièrement des actions découlant spécifiquement de la perte progressive d'autonomie ou de la maladie grave d'une personne leur étant liée par parenté ou affection; les situations de handicap peuvent également entrer dans ce champ, mais notre propos ne s'y rapporte pas ici. Nous n'en faisons donc pas un usage défini par le nombre d'heures effectuées par ces individus par semaine ni par le type des tâches accomplies. À noter enfin que les personnes concernées ne se reconnaissent guère dans ces dénominations, ou alors rétrospectivement, une fois que l'aide - pour différents motifs - a cessé. 
commencent pourtant à être l'objet de programmes de politiques publiques. Ces derniers sollicitent leur contribution tout en développant - encore timidement diverses formes de soutien financier, organisationnel, social ou psychologique.

En Suisse, mais plus généralement dans les pays du Nord, la cellule familiale devient l'un des piliers stratégiques - du point de vue des pouvoirs publics - pour répondre aux effets combinés du vieillissement de la population, de l'augmentation de l'espérance de vie et de la chronicisation des pathologies, ainsi qu'à la nécessité de maîtriser les coûts de la santé. Or, quels effets ces changements sociodémographiques produisent-ils dans la vie privée de ces familles, tout particulièrement lorsque ces dernières font face à la maladie grave ? Cette question a émergé dès la seconde moitié des années 1990 avec, notamment, les travaux de Cresson (1995) et, par la suite, ceux qui analysent l'invisibilité du travail familial (Trabut et Weber 2009) en mettant non seulement en perspective l'articulation entre intervention familiale et intervention professionnelle, mais aussi les tensions morales, affectives et matérielles auxquelles sont soumis les intervenants familiaux (Weber et al. 2003 ; Cresson 2006, 2014 ; Fobre 2010). D'autres travaux (Blanc 2010 ; Dumont et al. 2010 ; Perrig-Chiello et al. 2010 ; Jacobs et al. 2011) ont par ailleurs démontré que le motif de l'engagement au chevet d'un proche est socialement distribué en fonction du genre, des moyens financiers, du type d'activité professionnelle selon la marge de manœuvre qu'elle laisse ou encore de la distance géographique qui sépare le foyer du malade de celui des parents susceptibles de s'en occuper.

Depuis environ une décennie, bon nombre d'initiatives portées par les pouvoirs publics - par le moyen de dispositions légales reconnaissant le rôle et le statut des proches, de mesures de congés d'accompagnement ou de services de soutien aux proches aidants - visent une meilleure articulation entre le monde des soins, la sphère domestique et celle du travail. Toutes ces initiatives ont non seulement en commun le fait de désigner le domicile comme un lieu de prise en charge médicale, mais également d'institutionnaliser le rôle des proches en faisant - volontairement ou non - le pari que les « dissonances » (Ferreira 2003) relationnelles et affectives qui peuvent apparaître entre une personne malade et ses proches, ou encore entre les proches et leur propre environnement social, peuvent être atténuées dès lors que les proches aidants sont encadrés et accompagnés.

L'objectif de cet article est de mettre en perspective la façon dont le recours aux proches aidants par des politiques publiques diverses est mis à l'épreuve par l'espace intime des personnes concernées et ce qui s'y déploie au moment où surgit la maladie. La notion d' « intimité » - qui fait l'objet d'une attention sociologique désormais renouvelée (Berrebi-Hoffmann 2009 ; BerrebiHoffmann et Saint-Martin 2016) - renvoie communément à ce qui relève de la vie intérieure, des rapports de soi à soi. L'intime contient ce qui est censé rester caché, secret, ce qui se dérobe à l'observation externe et qui se partage avec parcimonie et précaution. L'intime dénote aussi la profondeur de la personne - 
son intériorité - et constitue la sphère où se décline la singularité d'un individu ou d'une relation. Ce terme renvoie donc également au fait d'être familier avec une personne, un lieu ou un objet.

Pour notre part, nous considérerons ici l'intime comme «l'espace qu'un individu dédie à soi et à un cercle restreint d'individus, où chacun peut cultiver sa singularité ou du moins l'idée qu'il s'en fait, en n'ayant a priori pas d'autres contraintes que celle imposée par ce cercle choisi ». Pourtant, lorsque la maladie apparaît de façon durable, que sa prise en charge impose un rythme, des gestes et des conséquences sur lesquelles les individus n'ont qu'une faible prise, cet espace s'avère lui aussi précaire. Ainsi, l'intime apparaît comme un espace ni complètement clos ni pleinement ouvert. Il devient ce lieu clivé, voire clivant, où se vivent des tensions et des contradictions d'autant plus fortes que les pouvoirs publics nourrissent des attentes à l'égard des proches aidants.

Pour illustrer empiriquement les contours d'une telle dynamique, nous prenons appui sur une enquête menée en Suisse romande (francophone), dont l'objectif était de saisir les tensions dans la conciliation de l'activité professionnelle et de l'accompagnement d'un proche atteint d'une maladie potentiellement fatale ${ }^{2}$. Nous focaliserons notre attention sur des situations dans lesquelles le rôle de proche aidant s'avère difficile à endosser, révélant de la sorte la nature des obstacles qui - issus de la sphère privée - ont une incidence directe sur le succès d'un projet politique.

\section{Dire et taire...}

Comment devient-on un proche aidant accompagnant un parent malade et comment articuler ce rôle avec une activité professionnelle ? À l'annonce du diagnostic d'une maladie grave, une temporalité indéterminée voit le jour, dans laquelle les engagements quotidiens, plus ou moins routiniers, plus ou moins

2. Cette recherche est intitulée Les "proches aidants": entre monde du travail et accompagnement de fin de vie. Elle a été financée par le Fonds national suisse de la recherche scientifique ( $\mathrm{n}^{\circ} 139246$ - septembre 2012-mai 2016) dans le cadre de son programme national de recherche Fin de vie (PNR67, www.pnr67.ch) et réalisée par les deux signataires de l'article, avec la collaboration de Nicole Brzak. Notre protocole de recherche a été validé par la Commission cantonale d'éthique de la recherche sur l'être humain (Vaud) en 2012. Nous avons mené une série d'entretiens semi-directifs approfondis d'une durée moyenne de deux heures $(41$ proches ; employés au minimum à $80 \%$ - ou à $60 \%$ avec enfants en bas âge durant toute la période de l'accompagnement ; qui se sont occupés ou qui s'occupaient, au moment de l'entretien, d'un parent ou d'un ami malade, souffrant du cancer dans la majorité des cas). Les personnes interviewées ont été recrutées par le biais de partenaires de terrain, tant dans le champ sociosanitaire (structures palliatives, centres de soutien aux proches, ligues contre le cancer) que dans le monde du travail (entreprises privées et publiques). Lorsque la situation s'y prêtait, et avec leur accord, nous avons également rencontré leurs collègues et supérieurs hiérarchiques (14 entretiens). Dans quelques cas (trois entretiens), nous avons également mené une interview avec la personne malade elle-même. Pour plus de détails sur le dispositif de recherche, voir Berthod et al. (2017). 
réglés, sont remis en question. Les parents, et tout particulièrement les femmes, ne gèrent plus des journées à double tâche - travail rémunéré et gestion du foyer -, mais à triple tâche puisque s'y ajoutent des déplacements fréquents à l'hôpital, de nombreux soins ou l'accomplissement de tâches logistiques. Tout ceci s'ajoute à l'inquiétude que peut susciter le diagnostic ou le protocole thérapeutique qui en découle. Dans ces situations, le temps disponible semble toujours trop réduit : est-il possible d'assister aux différents rendez-vous médicaux et d'assurer une présence ordinaire au travail ? De privilégier une carrière professionnelle en mobilisant des tiers pour prendre soin d'un parent dont l'espérance de vie est incertaine ? Dans le domaine des sciences sociales, la littérature scientifique sur l'aide informelle fournie par les proches dans les pays du Nord le montre bien : le mode de conciliation de la sphère professionnelle et de la sphère domestique influence non seulement le rôle des membres de la famille et des personnes qui vivent sous un même toit, mais aussi : 1) les relations qui se tissent entre elles, y compris au travail (Berthod et al. 2016 ; Hochschild 1997 ; Kröger et Sipilä 2005 ; Le Borgne-Uguen et Rebourg 2012 ; Pailhé et Solaz 2009) ; 2) la manière dont les proches remettent en question leur propre façon de mener leur vie (Papadaniel et al. 2017).

Lorsque la maladie surgit dans leur entourage, les proches passent beaucoup de temps à trier l'information qu'ils souhaitent faire circuler dans leur milieu de travail, selon les moments et selon les interlocuteurs. Nous avons ainsi observé que l'annonce de la maladie d'un proche ne va pas de soi. À qui l'annoncer et comment le faire sont des questions qui se posent d'entrée de jeu et qui ne trouvent jamais vraiment de réponse définitive, révélant ainsi les difficultés incessantes auxquelles les proches font face par rapport à leurs collègues, leurs prestataires ou leurs clients. Communiquer, de surcroît, au sujet de la maladie grave d'un proche suppose un accompagnement conséquent, ce qui conduit potentiellement à se voir assigner le rôle de proche aidant, ce qui ne va pas de soi non plus.

Nous pouvons illustrer cette situation par l'exemple de Michel. Ce dernier a une quarantaine d'années ; il est marié et père de deux enfants de moins de dix ans. Sa mère est tombée malade du cancer soudainement. Après un premier cycle de chimiothérapie bien supporté, elle subit une récidive quelques mois plus tard. Elle fit quelques allers-retours entre son domicile et l'hôpital, où elle séjourna un mois en soins palliatifs, jusqu'à son décès à la fin de l'année 2013. Sa maladie aura duré environ une année et demie. Cette période a été marquée par une grande solidarité familiale. Le beau-père de Michel était un soutien au quotidien pour sa mère. Son frère, habitant la même ville, s'était également engagé auprès de sa mère ; sa tante apportait un soutien par le biais d'une aideménagère ponctuelle. L'organisation des efforts était ainsi bien réglée par ces proches. Des services professionnels intervenaient en cas de besoin, avec plus 
d'intensité durant la phase terminale de la maladie. L'information sur la santé de sa mère passait par Michel, qui était en première ligne et constituait un pivot de l'organisation de la solidarité familiale.

Michel travaille comme avocat dans une petite étude. Il est le bras droit de l'associé principal, son chef. Deux secrétaires complètent l'équipe. Bien qu'il ait obtenu le soutien explicite de ses supérieurs hiérarchiques, Michel a poursuivi ses activités professionnelles en tentant d'honorer au mieux de ses possibilités ses rendez-vous et de respecter les délais des différents dossiers qu'il avait à sa charge. Il nous décrit ainsi son organisation :

Disons que ça m'a fait un gros coup sur le moral. C'est important, ça s'ajoute aux difficultés. Mais cela n'a pas eu d'implication immédiate, si ce n'est quelques téléphones occasionnellement ou des demi-journées d'absence. Au début, ça n'a pas été une grosse implication. Mais après, oui ! J'ai vu mes capacités de travail diminuer, avec un impact clair sur le travail ; j'ai dû prendre des antidépresseurs pour tenir le coup. [...] Le dernier mois... les derniers mois ont été terribles. L'impact sur mes capacités de travail était évident. Je n'arrivais plus vraiment à me concentrer, à faire tout ce que je devais faire. En même temps, les dossiers étaient là et il fallait bien faire tourner l'étude.

Barbara, la secrétaire de Michel, que nous avons rencontrée, dit avoir été informée de sa situation dès le début. Mais, en dehors d'un cercle rapproché, elle souligne que Michel restait discret sur sa situation et qu'il n'en parlait, par exemple, jamais avec ses clients. À ses yeux, Michel voulait montrer qu'il tenait le coup, qu'il « gardait la tête hors de l'eau ». Elle observait sa baisse de concentration et notait bien la surcharge : « Il fuyait dans le travail ; quelque part, ça l'arrangeait. » Personne au sein de l'étude n'était d'ailleurs au courant que Michel prenait des médicaments, une information lâchée en fin d'entretien, sur le ton de la confidence.

Le lieu de travail n'est pas l'endroit où l'on souhaite divulguer les secrets de sa vie familiale et privée, et encore moins parler de la manière dont on les vit. Cette tension entre sphère intime et sphère professionnelle est d'autant plus sensible que l'enjeu n'a pas uniquement trait au maintien du salaire. En Suisse, mais plus généralement en Occident, autant la maladie du proche apparaît comme un facteur désocialisant, autant le travail ressort comme un moyen de préserver son rang et son identité sociale. En créant des coupures dans la relation avec le malade, en obligeant le proche à se concentrer sur sa tâche, en lui faisant quitter le domicile, le travail assure cette ouverture sur l'extérieur et l'accès à des éléments détachés de la maladie ou du rôle d'accompagnant. Mais, en retour, il peut faire émerger des difficultés. Celles-ci sont particulièrement visibles dans la situation de Gérôme, employé d'une compagnie d'assurances, dont l'épouse est atteinte d'un cancer : 
GÉRôME - Mais je me concentre et puis, j'sais pas, encore lundi j'ai fait une boulette : je devais mettre une date, j'sais pas, le 20 octobre - je mets le 30 septembre. Pourtant, je me concentre : je sais que je dois changer la date ; je fais faux. Pis après, ça s'est enchaîné toute la journée : je rectifiais, je faisais faux, enfin. [...] Un dossier qui devait être traité en une demiheure, j'ai mis toute la journée. Et là, je me suis rendu compte, je me dis : « Ça va plus. On peut plus continuer comme ça. » Alors, bon, mon chef, je l'ai vu hier, pis il m'a fait aussi la remarque, il m'a dit : " T'as passé toute la journée là-dessus. » Pis, je lui ai dit... Donc, il avait vu. [...]

INTERVIEWER - Comment est-ce qu'il sait que vous avez passé toute la journée là-dessus ? C'est parce que...

G. - Parce que je lui ai posé 15000 questions dans la journée, pis des questions, je dirais, à la con parce que... c'est un petit peu aussi [une] perte de confiance en moi. Euh, en plus, hier, elle [sa femme] allait à Genève, pour faire un[e] IRM, enfin tout un contrôle qu'elle fait tous les deux-trois mois. Puis, elle ne voulait pas y aller, pis elle était limite. Bon, c'est pas douloureux, mais ça pèse, maintenant, d'y aller - donc, voilà. Alors, ça c'est bien passé, etc. Donc, moi, en travaillant, je pensais aussi ce machin, enfin, c'est... Je ne suis plus à $100 \%$ au boulot, quoi.

Maintenir une activité professionnelle se fait de manière incertaine et tâtonnante, laissant potentiellement le travailleur exposé en cas de baisse de productivité ou de relations flottantes avec les collègues. De nombreux salariés évoquent l'impression d'être en situation constante de porte-à-faux : au travail, le proche laissé seul fait l'objet d'un souci ; au domicile, le travail accompli trop vite est facteur d'anxiété. Ou encore est-il demandé aux collègues que l'on a mis au courant de la situation de ne pas faire trop souvent référence au proche malade. Les circonstances exigent simultanément une confiance et un certain relâchement dans un équilibre assez délicat. Ainsi, Flavie, employée d'une grande entreprise dont le père est décédé d'un cancer quelques mois avant l'entretien, nous déclare :

Je leur avais dit $[\ldots]:$ : "Moi, je ne suis pas une pestiférée, ici. Je veux pas que vous veniez me dire : "Hein, mais ça va ?", toutes les secondes. Ça m’énerve. Donc, on fait comme avant. Si je me mets à pleurer, bah, vous allez, voilà, là-bas. » Et puis, ils ont dit : « Non, pas de problème » et ils se sont comportés comme avant avec moi. Et si, moi, je voulais leur parler, eh bien, je parlais.

Cette régulation du partage et la définition des conditions sous lesquelles un individu évoque un événement privé dans une sphère qui ne l'est pas donnent à voir les débordements contradictoires de l'intime. En ouverture du deuxième tome de L'histoire de la vie privée, Georges Duby (1999) avertit ainsi le lecteur que la façon de traiter cet objet est en fait conditionnée par la masse et le type des données disponibles. Dans la mesure où l'intime renvoie au caché ou à ce qui n'est partagé qu'avec parcimonie, son analyse implique toujours une rupture avec sa logique. 
Celle-ci n'est pas toujours possible ou complète. L'accès à un matériau intime - un journal, des lettres, une confidence - peut s'avérer aléatoire ou incomplet, comme le souligne ailleurs Catherine Deschamps : « chez une même personne, la variabilité de l'exposition selon le retour escompté rend caduque toute entreprise de compréhension de l'intimité fondée sur une source unique » (2008: 139). Ce qui est valable pour les chercheurs l'est également dans l'action ainsi que pour les acteurs. Michel, Gérome ou Flavie livrent à leurs collègues des informations qui excèdent leurs rapports professionnels soit malgré eux (parce qu'on observe leurs attitudes, leur tenue, leur apparence ou leur performance) soit au prix de requêtes paradoxales ( «Faites comme si de rien n'était, mais soyez prêts à m'écouter ») qui se distinguent des règles usuelles d'interaction entre collègues. Une telle posture fait indirectement écho aux travaux de Veena Das (2006) sur le partage d'une expérience sensible qui ne trouve sa place dans aucune pratique d'écoute ou de partage existante. L'on pourrait penser que cette régulation « toujours à faire " ou au « cas par cas » ne se rencontre que dans la superposition d'ordres sociaux inconciliables. Les échanges prendraient alors une tournure plus réglée dans la sphère privée, là où - précisément - le partage est censé aller de soi et où il peut faire l'objet d'ajustements ; là où le rôle de proche aidant paraît plus évident à endosser. La situation de Marion et de Gérald et puis celle de Clara et de Paul nous montreront que la sphère privée n'est pas exempte d'adaptation, voire de profonds malentendus.

\section{Seule malgré elle}

Marion et son époux Gérald, décédé trois mois avant notre entretien, ont été confrontés à la maladie par surprise, voire par erreur. Ce faux départ s'explique par un vraisemblable manque de coordination entre les structures de soins par lesquelles le couple est passé. Marion et Gérald vivaient dans une région périphérique. Souffrant d'une toux chronique et aiguë qui le poussait régulièrement à faire des syncopes, Gérald finit par consulter son médecin généraliste. Ce dernier, aux dires de Marion, «a vu une glande qui était déformée, qui avait grossi » non loin du poumon. Il a alors dirigé son patient vers un chirurgien de l'hôpital régional le plus proche de son domicile, sans autre indication. Le couple ne rencontrera jamais ce dernier, qui transférera automatiquement le dossier de Gérald à l'hôpital universitaire situé 50 kilomètres plus loin :

On a reçu un [appel] téléphon[ique] [...] on devait se présenter au CHU tel jour, à telle heure, chez un chirurgien. Quand on est arrivé au CHU, le chirurgien nous a reçus et il nous a dit : "Vous voulez que je fasse quoi ? C'est une tumeur, je ne peux rien faire. » C'est là qu'on a appris.

L'entrée dans la maladie a donc été brutale. Associée à la gravité du diagnostic, cette brutalité a entrainé un effet de sidération chez Marion. Après la pose d'un stend pour atténuer la contraction d'une veine cave due à la tumeur, les soins se sont organisés dans les structures régionales. Un protocole 
de chimiothérapie a été mis sur pied. Une circonstance aggravante s'est alors présentée, d'autant plus incompréhensible pour Marion que sa relation avec Gérald ne battait pas de l'aile :

Mon mari ne voulait pas que je participe à sa maladie, donc je n'ai pas pu l'accompagner en chimio. Il ne voulait pas que je l'accompagne chez les médecins quand il avait des scanneurs ou des résultats. Et j'ai dû gérer un peu ça avec mon travail à $100 \%$. L'angoisse. Et les informations ne venaient que de lui.

Rien n'obligeait formellement Gérald à inclure sa compagne dans le processus de soins. Marion continuait néanmoins à se tenir aux côtés de son époux, notamment lorsqu'il était hospitalisé. Les interventions du personnel soignant, principalement celles des médecins, faisaient apparaître de plus en plus clairement une zone de non-dit entre Marion et Gérald. Les médecins la questionnaient sur les éventuelles directives médicales pour lesquelles opterait Gérald, mais elle était incapable de répondre. Par ailleurs, les médecins tentaient de la préparer à l'inéluctable, mais elle se montrait plutôt fermée. Cette fermeture s'explique par le fait que Marion n'avait pu participer à aucun des rendez-vous médicaux où il était question de faire le bilan d'un traitement, et donc de la régression ou progression de la tumeur : « J'avais rien », nous a-t-elle dit. La vie à la maison était devenue compliquée :

On n'avait plus aucun sujet de discussion ; on avait comme une troisième personne qui était au milieu. Il y avait la maladie. Lui, il passait ses journées ici, à la maison ; il allait au village voir des copains. Moi, c'était le boulot et la maison. Il n'y avait plus vraiment de sujet à discuter, le soir. Et on ne pouvait pas parler de la maladie.

Il se fâchait, il se fermait complètement. [...] Lui, il voulait que les gens soient normaux comme s'il n'y avait pas eu de maladie [...]. Moi, je ne me sentais pas bien ; pas bien parce que, pour finir, on se parle peu, quasiment plus. Pour moi, c'était un travail dans la tête, j'avais plein de questions que je n'osais pas demander [sic].

Son activité professionnelle lui offrait la possibilité de sortir de cette impasse, mais celle-ci n'était pas suffisante pour contrer le malaise grandissant : « On ne partage pas ce genre de choses avec ses chefs. » Marion n'avait donc aucun interlocuteur avec qui partager son expérience. La mise en commun était bloquée par un double effet de contrainte : le premier tenait à l'impossibilité d'aborder ouvertement la maladie avec son conjoint; le second renvoyait à une loyauté en regard des consignes de la personne malade. Cette loyauté pouvait se démultiplier. Par exemple, Gérald ne s'est que peu confié à son épouse, mais il a brièvement évoqué son expérience avec quelques amis : "Certaines personnes 
ont discuté avec lui, mais avaient beaucoup de peine à me le dire, car elles savaient qu'il ne me parlait pas - parce que je pense qu'il leur a dit qu'il ne le voulait $\operatorname{pas}^{3} \gg$.

C'est précisément pour ne pas s'ingérer dans la sphère du couple que les amis sollicités par Gérald n'ont pas transmis d'informations à Marion : le respect de la demande de Gérald de ne pas partager son expérience de malade a donc créé l'isolement progressif de sa femme. Seule face à elle-même, Marion ne s'en remettait qu'à des questions auxquelles elle ne pouvait répondre que très partiellement :

C'est venu par petites bribes, mais je pense que je ne sais encore pas tout. Mais je sais qu'il se savait perdu [...] depuis le premier entretien, depuis la première chimio... Je pense ! C'est ce que je pense maintenant ! Mais j'ai pas non plus la preuve que...

Ce que j'aimerais savoir maintenant, [c'est] si lui, il en a souffert. (Elle pleure.) C'est le point le plus difficile. Du fait que je ne suis pas arrivée à gérer la situation [...]. Non, mais par rapport à lui, est-ce que j'aurais pu le soulager en étant... bah, justement... en n'étant pas que focalisée sur la maladie et en essayant d'avoir été plus... je sais pas.

Selon Marion, il lui a manqué un interlocuteur, mais également un savoirfaire pour gérer la distance que la maladie a placée entre elle et son conjoint. Elle se réfère alors à une abstraction ( $\mathrm{Il}$ y a des gens qui arrivent à être normaux ») ou à un interlocuteur silencieux, son chat, dont le statut révèle précisément tout son désarroi ${ }^{4}$ : « Le chat m'a fait beaucoup de bien [...]. Il amenait quelque chose. On peut leur causer, à eux [aux animaux]. Ils ne parlent pas, mais ils sont beaucoup [sic] présents. »)

Les questions sans réponse ou les réponses tâtonnantes que tente d'apporter Marion donnent à voir l'architecture contrainte de son espace intime. Au travail, à la maison, face à ses amis, il lui manque un point de référence pour statuer sur ce qui leur est arrivé, à elle et son mari. Lorsque les conditions du partage au-delà des seuls déterminismes liés au genre ou aux moyens économiques - ne sont pas réunies, l'intime se transforme en une zone de solitude et d'inconfort.

\section{L'épreuve de la maladie et la singularité des trajectoires}

Soutenir une personne gravement malade pour laquelle on éprouve une forte affection relèverait d'une posture vertueuse faite de loyauté et d'amour, comme en témoignent diverses campagnes de services de santé publique. Cellesci visent à reconnaître l'engagement des proches, notamment en les remerciant par le moyen de l'affichage public ou dans la documentation leur étant destinée.

3. Pour ne pas l'inquiéter (note des auteurs).

4. Le rôle des animaux domestiques n'est pas à sous-estimer, à tel point d'ailleurs qu'ils font l'objet d'une attention médicale (voir Brooks et al. 2016). 
C'est à partir de cette norme idéalisée que prospère l'image d'une prise en charge à domicile répondant à la volonté des patients et à celle des pouvoirs publics pour éviter des hospitalisations trop longues et pallier la pénurie de lits (Ginn 2013). Cet idéal se déploie pourtant dans un régime hybride où le projet politique investit la sphère intime. Non seulement ce projet brouille la frontière entre des prises en charge formelles et informelles, mais il est susceptible de créer des troubles d'autant plus forts que les proches ne peuvent invoquer la distance professionnelle et ses impératifs. La personne malade et ses attentes participent de l'équilibre de la démarche. Flavie a ainsi pu accompagner son père dans une relative sérénité, car celui-ci s'est contenté de son sort sans exiger qu'elle donne davantage. À l'inverse, Gérôme a essuyé les reproches de son épouse :

$\mathrm{Y}$ a plein de choses qui reviennent, qui sortent, pis bon, à chacun notre vécu, donc. À force de la mettre dessous, à un moment donné, ça pète [...]. Ah, il y a eu des tensions, il y a eu d'énormes tensions. Elle m'a beaucoup reproché de... de... euh... quand elle a fait les rayons, la chimio... de ne pas être vers $[s i c]$ elle, de ne pas [m']occuper d'elle. Alors, peut-être que je n'ai pas été aussi présent qu'elle le voulait, mais je devais gérer tout le reste, quoi. Et les filles, ben voilà : les filles, ça bougeait dans tous les sens, quoi, donc... Moi, j'essayais de concilier au mieux. Maintenant j'ai fait au mieux, et pour elle... elle me reproche encore maintenant que je ne me suis pas occupé d'elle. Surtout, quand il y avait les chimios où, pendant quatre-cinq jours, elle [était] vraiment mal. Et je devais être tout le temps à côté d'elle. Et je n'arrivais pas... ben voilà, quoi, je veux dire, il y avait le reste qui bougeait, qui continuait à tourner, quoi - donc, c'était pas évident.

Si l'on considère l'intime - en prenant pour point de référence les sociétés des pays industrialisés ${ }^{5}$ - comme l'espace qu'un individu dédie à lui-même, où il peut cultiver sa singularité ou l'idée qu'il s'en fait (Giddens 1991, 1992, 1993), à l'abri de la contrainte sociale et sous le regard bienveillant de son entourage le plus proche, on se rend compte par les exemples de Flavie, Gérôme et Marion que la géométrie de cet espace est fluctuante. Michelle Perrot (2009) a d'ailleurs illustré ces fluctuations de l'intime en analysant la chambre à coucher à travers les époques en tant que lieu d'un retrait où un individu peut cultiver un dialogue, avec lui-même ou les autres, libéré de la contrainte sociale. Mais l'historienne souligne bien que ce même espace de la chambre ne se résume pas à cette liberté de disposer de soi : il est un lieu possible de spectacle - le coucher du roi en est l'exemple paradigmatique ${ }^{6}-$, mais aussi d'enfermement ou de confinement. Si

5. Ceci ne signifie pas pour autant que l'Occident industrialisé ait le monopole de l'individu et du retour sur soi. Pour une synthèse, voir Laé (2003), Vigarello (2014) ou Lozerand (2015).

6. Dans une perspective sociologique, on pourra se référer aux réflexions de Marc Loriol (2016) sur le travail diplomatique et la façon dont il implique une confusion entre sphères politique, professionnelle, privée et intime. Cet article, en français, fait écho, notamment, à un article plus ancien d'Arlie Hochschild (1969) et à un ouvrage de Katherine Hughes (1999) sur le rôle d'épouse d'ambassadeur. 
la promotion publique du rôle des proches est l'équivalent du coucher du roi, il faut se rappeler que cette fonction peut être contraignante, voire aliénante. On ne se départit pas facilement de l'intime et de ses équivoques, entre liberté et isolement.

C'est ce que démontre encore avec d'autres nuances l'exemple de Clara. Cette dernière est âgée d'une cinquantaine d'années et travaille à temps partiel dans l'enseignement. En 2012, les médecins découvrent chez Paul, 64 ans, son époux, un mélanome oculaire dont il décèdera trois ans plus tard. Les deux conjoints ont rapidement compris l'agressivité de la maladie, alors que Paul était en parfaite santé jusqu'à ce diagnostic : " Je pleurais tout le temps ; j'ai vu tout de suite la gravité. Lui ne s'est jamais intéressé à sa maladie. Ce n'était pas un sujet de conflit. "

$\mathrm{Au}$ travail, Clara peut se contenter de communiquer avec sa direction et obtient l'accord de celle-ci pour s'absenter lors des rendez-vous médicaux. Ce motif n'était pas toujours vrai, car elle s'en servait aussi pour être simplement présente auprès de son mari. Son employeur, " extrêmement humain et intelligent ", ne posera aucun obstacle. Malgré cela, la première année au travail s'avère compliquée. Clara fait face à beaucoup d'angoisses et a de la peine à se concentrer ; elle anticipe sans cesse les difficultés, surveillant les moindres soubresauts de la maladie : «J'étais obnubilée par la maladie. » Deux ans plus tard, des métastases apparaissent. La situation devient plus difficile à vivre pour Clara, en particulier la gestion de ses activités professionnelles. Sa thérapeute décide de la mettre en arrêt de travail durant six mois pour éviter des aller-retour aléatoires vis-à-vis des élèves. Durant cette période, elle est amenée à rencontrer, par le biais de son employeur, un médecin-conseil qui ne prend pas toute la mesure de son statut de proche. Clara le juge inadéquat :

Quand on est en arrêt de travail, on a vraiment besoin d'être conseillée. J'ai extrêmement mal vécu cet arrêt alors que Paul travaillait, que mon fils allait à l'école et que moi, je restais chez moi. Je me disais que c'est injuste : « Je ne peux pas enseigner, mais je peux faire d'autres choses. » Le fait d'être payée à ne rien faire et de devoir accepter ma fragilité, pour moi, c'était extrêmement difficile. J'attendais de la part du médecin-conseil qu'il me propose quelque chose, qu'il me fasse des propositions, et je n'ai rien eu. J'ai eu une espèce de contrôleur, qui s'assurait que je n'usurpais pas le statut de malade.

Cette intervention médicale, en lien avec l'activité professionnelle, influence l'épreuve que représente la maladie d'un être cher. En l'occurrence, elle n'a pas favorisé la préservation des liens sociaux de Clara; au contraire, elle a non seulement participé au confinement de cette expérience dans une sorte de singularité - cela, d'autant plus que son conjoint malade continuait de travailler -, mais aussi au repli de Clara sur la seule sphère personnelle. Ce n'est que dans 
un deuxième temps que Clara reprendra son activité professionnelle et elle la considérera comme une véritable ressource durant toute la dernière année de vie de son conjoint.

Parallèlement, il est à noter que le rapport au monde médical s'est construit en lien avec l'accompagnement de Paul lors des visites médicales et du suivi des traitements : " J'ai voulu rencontrer le médecin ; Paul n'a pas voulu que je l'accompagne. Il a appris qu'il en avait encore pour une année environ. [...] Il n'y avait pas de soin particulier à [donner]. Il m'a dit : “Tu es ma femme et pas mon infirmière". » Elle demandait que Paul informe leur fils ; c'était douloureux pour lui de le faire : "Paul voulait connaître les risques et bénéficier du traitement. Il voulait juste continuer une vie normale le plus longtemps possible. » Clara, de son côté, fait appel à un groupe de soutien associatif de la région : "Quand j'ai appelé, j'ai eu le sentiment d'être enfermée encore plus dans la maladie. Je ne l'ai pas vue de manière positive, cette affaire-là. »

Son avis sur les groupes de parole est radical : "C'est comme un corporatisme, être dans un groupe de cancéreux. La maladie vous fige dans l'identité de malade ; et aller là-dedans, ça accentue encore plus ça. » Clara dit alors les efforts, déployés par son mari et elle, pour, précisément, ne pas être affectés à la maladie. Cela semblait plus facile pour Paul, qui est même devenu une ressource pour elle durant la deuxième phase de la maladie :

À l'annonce du mélanome, en 2012, j'ai eu toutes les angoisses de ma vie : je me suis projetée. Après, j'ai vraiment découvert, comme on le dit en littérature - j'ai fait des études littéraires -, le ici et maintenant. Paul m'a aidée à vivre l'ici et le maintenant. Je n'avais aucune envie d'anticiper la mort.

Clara allait voir le médecin avec une batterie de questions ; Paul ne venait pas toujours. Elle avait besoin de comprendre la situation. Face aux réponses et aux nouvelles informations, il lui était difficile de savoir si Paul était angoissé ou pas. Clara qualifie son mari, méfiant en outre à l'égard des médecins, de « taiseux » : « Je ne sais pas comment il l'a vécu. » Durant les consultations médicales, il écoutait les réponses, mais n'en demandait pas trop :

Une fois, on a eu un clash dans le bureau du médecin. J'avais été trop [loin] - qu'est-ce que j'avais demandé au médecin ? Je ne suis plus sûre. Mais Paul a réagi à mes questions en se désolidarisant de moi. Et moi, je l'avais extrêmement mal perçu et ressenti. [...] En gros, il me demandait de me taire. [...] Ça s'est passé assez tôt après l'annonce des métastases. Mais ça a eu l'effet, je dirais, d'un assainissement de notre histoire ; on était jusque-là un couple aimant. Il y avait des choses qui ne fonctionnaient pas, mais avec lesquelles je m'accommodais. Mais là, je lui ai dit : « Pour t'accompagner, j'ai besoin d'être dans le vrai et je ne peux plus m'accommoder [...]. Moi, j'ai besoin de poser toutes les questions que je veux. » 
Cette posture a rencontré, avec l'accord de Paul, la bienveillance des médecins traitants qui ont dès lors accueilli Clara pour aborder avec elle la situation de son mari, dans un dispositif de prise en charge inhabituel :

J'ai toujours senti que j'avais entièrement ma place auprès des médecins ; je ne me suis jamais sentie [comme] l'intruse. Comme j'avais beaucoup de questions à poser et que Paul ne supportait pas ça, je demandais des entrevues seule avec le médecin. Il y a deux manières de vivre la maladie. Le médecin a dit : «Écoutez, ce n'est pas courant, mais pourquoi pas ?

\section{Conclusion : équivoques de l'intime et sollicitations des politiques publiques}

Clara estime avoir formé avec Paul un couple soudé ; elle décrit une relation d'amour et de confiance présente jusqu'au décès ; la maladie les a néanmoins bousculés et est venue se placer entre eux en les exposant à un risque d'isolement « existentiel » résultant du mouvement de « désolidarisation » qu'évoque Clara. Ce mouvement souligne la différence inéluctable des trajectoires qu'empruntent les deux membres du couple, ce que Gérôme, Marion et, dans une autre configuration, Flavie et Michel (puisqu'il s'agissait respectivement de la maladie du père et de la mère) ont tous éprouvé avec une certaine intensité. Cette différence des trajectoires est survenue malgré eux, dans la mesure où tous ces proches ont souligné la solidarité - qu'elle soit effective ou non - qui prévalait avant ou qui a continué durant l'accompagnement de la personne malade.

L'intime échappe ici à un strict rapport de soi à soi. Il ne correspond pas à un travail sur la « profondeur » du soi ou à la découverte de sa « singularité »; il ne se limite pas non plus à la familiarisation avec une situation, la maladie, notamment. Les frontières de cet espace intime deviennent au contraire floues, voire poreuses; elles révèlent en clair-obscur le fait que cet espace est socialement et culturellement construit. Cette dilution de l'architecture de l'intime se révèle dans une suite d'interrogations aux réponses très aléatoires et peu codifiées : comment se conduire ? Comment accompagner ? Que faut-il chercher à savoir ? Faut-il intervenir, tout dire, ne pas dire ou se tenir à distance ? Cet espace intime apparaît alors comme ambigu, voire incertain. Il donne de ce fait difficilement prise aux initiatives étatiques et publiques qui témoignent de la reconnaissance envers les proches et cherchent à soutenir ces derniers sur le plan émotionnel et psychologique, notamment.

Ces questions qui envahissent cette dimension intime des proches sont non seulement délicates parce qu'elles mettent en jeu la maladie et ses conséquences, mais aussi parce que les uns et les autres peuvent y répondre de façon radicalement opposée. Chaque action et chaque prise de parole sont susceptibles d'alimenter les doutes, de nourrir les non-dits, de donner lieu à une surenchère d'hypothèses et, dès lors, de conduire à une rupture qui ne dit pas tout à fait son nom. Et ces questions ne sont pas nécessairement liées à la 
possibilité de bénéficier de congés d'accompagnement (une disposition légale qui est en discussion, mais qui n'existe pas encore en Suisse sur le plan fédéral), à un soutien financier ou à un lieu d'écoute pour exprimer son ressenti. Elles relèvent plutôt de normes sociales et culturelles propres à la maladie et au rapport à la mort ainsi qu'à une pédagogie plus globale de l'accompagnement qui dépassent bien souvent les programmes ciblés sur les individus, sur les proches aidants.

La situation de Clara en fournit une bonne illustration : le monde du travail reste encore trop prompt à interpréter la situation des proches en termes psychosociaux, avec le risque de renforcer le sentiment de confinement du statut de proche dans la maladie. Et les groupes de parole entre pairs ne répondent pas à l'ensemble de ces enjeux. Le caractère " corporatiste » que leur prête Clara renvoie à l'affectation que cache la catégorie de proche aidant, dont on ne sait si elle est définie à partir des contraintes liées à la maladie ou à celles du modèle de loyauté parentale qu'elle induit. Une telle ambivalence - ressentie par la grande majorité des proches que nous avons rencontrés - renvoie à la cohabitation, face à la maladie d'un être aimé, de paramètres très différents : les attitudes respectives face à la maladie ; la possibilité de les articuler ; le lien avec le parent malade; ses attentes et les moyens à disposition pour y faire face ; les attentes face au personnel soignant ; l'aptitude des professionnels à y répondre ; le rapport au travail et la marge d'indépendance laissée aux individus dans le monde professionnel. En somme, chacun de ces paramètres nécessite de nouveaux ajustements à la lumière des éléments et attitudes attendus qui pèsent actuellement sur les proches.

Paul et Clara, de manière plus emblématique encore que dans les autres situations que nous avons décrites, ont été en mesure " de se mettre d'accord » par rapport à leurs différentes attentes face à la maladie et à ses inconnues. Ils ont pu, partiellement du moins, mutualiser leurs expériences respectives et, ainsi, gérer l'épreuve qui s'est introduite dans leur couple. À cet effet, le rôle joué par les médecins s'est révélé décisif puisqu'en livrant à Clara - dans un dispositif assez inédit - les informations relatives à Paul en son absence, ceux-ci ont servi, sans le savoir peut-être, de régulateurs de leur relation. C'est l'enjeu auquel sont confrontés les pouvoirs publics suisses en se penchant sur la catégorie des proches aidants qui se trouve ici résumée : trouver les leviers qui permettent, dans chaque situation, de soutenir les proches sans associer leur vie intime à une conception unique de la maladie et de la parenté, afin d'éviter leur isolement. Pour y parvenir, il importe finalement de ne pas centrer toute l'attention sur la figure du proche aidant (et sur sa relation avec la personne malade), mais de travailler aussi - et peut-être surtout - sur les modes de socialisation des collectivités face aux enjeux sociodémographiques contemporains dans une pluralité de contextes : écoles, entreprises, milieux associatifs, institutions sociosanitaires. Ce travail ne fait que commencer. 


\section{Références}

Berrebi-Hoffmann I. (dir.), 2009, Les politiques de l'intime. Des utopies sociales d'hier aux mondes du travail d'aujourd'hui. Paris, La Découverte.

Berrebi-Hoffmann I. et A. Saint-Martin (dir.), 2016, dossier « Dynamiques de l'intime », Socio. La nouvelle revue de sciences sociales, 7, consulté sur Internet (https://socio.revues. org/2361), le 25 mars 2019.

Berthod M.-A., Y. Papadaniel et N. Brzak, 2016, « Entre nous, le cancer : monde du travail et dynamiques familiales autour de l'enfant gravement malade », Enfances Familles Générations, 24, consulté sur Internet (https://journals.openedition.org/efg/966), le 25 mars 2019.

—, 2017, Les "proches aidants ». Entre monde du travail et accompagnement de fin de vie. Lausanne, Haute école de travail social et de la santé, consulté sur Internet (https:// www.eesp.ch/fileadmin/user_upload/rad/recherche/Rapports/170118_Rapport_PNR67sho88pCouv.pdf), le 25 mars 2019.

Blanc A. (dir.), 2010. Les aidants familiaux. Grenoble, Presses universitaires de Grenoble.

Brooks H., K. Rushton, S. Walker, K. Lovell et A. Rogers, 2016, « Ontological Security and Connectivity in an Uncertain World: A Study of Pets in the Management of the Everyday Lives of People Diagnosed with a Long-Term Mental Health Condition », BMC Psychiatry, 16, 409, consulté sur Internet, (https://bmcpsychiatry.biomedcentral.com/ articles/10.1186/s12888-016-1111-3), le 25 mars 2019.

Cresson G., 1995, Le travail domestique de santé. Paris, L'Harmattan.

—, 2006, «La production familiale de soins et de santé. La prise en compte tardive et inachevée d'une participation essentielle », Recherches familiales, 1, 3:6-15, consulté sur Internet (https://www.cairn.info/revue-recherches-familiales-2006-1-page-6. htm?contenu=resume ; DOI : 10.3917/rf.003.0006), le 22 septembre 2019.

—, 2014, « Les rôles et les tâches multiples de l'entourage des patients », Sciences sociales et santé, 32, $1:$ :65-70, consulté sur Internet (https://www.cairn.info/revue-sciencessociales-et-sante-2014-1-page-65.htm?contenu=resume ; DOI : 10.3917/sss.321.0065), le 22 septembre 2019.

Das V., 2006, Life and Words Violence and the Descent into the Ordinary. London, Berkeley, University of California Press.

Deschamps C., 2008, « Dits publics et écrits privés : représentations, médiatisations et confidences sexuelles », Sociologie et sociétés, 40, 2 : 131-145.

Duby G., 1999, « Avertissement » : 11, in P. Ariès et G. Duby (dir.), Histoire de la vie privée, tome 2. Paris, Seuil.

Dumont S., P. Jacobs, V. Turcotte, D. Anderson et F. Harel, 2010, « Measurement Challenges of In-Formal Caregiving: A Novel Measurement Method Applied to a Cohort of Palliative Care Patients », Social Science and Medicine, 71, 10 : 1890-1895.

FERreira C., 2003, «L'intimité familiale : quêtes et limites de l'engagement envers le proche », Sociologie et sociétés, 35, 2 : 149-161. 
Fobre N., 2010, The Invisible Heart: Economics and Family Values. New York, The New Press.

Fonds nAtional SuisSe De La ReCherche SCIEnTIFIQue, Programme national de recherche "Fin de vie " (PNR 67), consulté sur Internet (http://www.pnr67.ch/fr), le 16 octobre 2019.

Giddens A., 1991, Modernity and Self-Identity. Cambridge, Polity Press.

—, 1992, The Transformation of Intimacy: Sexuality, Love and Eroticism in Modern Societies. Stanford, Stanford University Press.

—, 1993, « Identité de soi, transformation de l'intimité et démocratisation de la vie » : 455-495, in M. Audet et H. Bouchikhi (dir.), Structuration du social et modernité avancée. Autour des travaux d'Anthony Giddens. Sainte-Foy, Les Presses de l'Université Laval.

GINN J., 2013, « Austerity and Inequality », Research on Ageing and Social Policy, 1, 1 : $28-53$.

Hochschild A., 1969, « The Role of the Ambassador's Wife: An Exploratory Study », Journal of Marriage and Family, 31, $1: 73-87$.

—, 1997, The Time Bind. When Work Becomes Home and Home Becomes Work. New York, Metropolitan Books.

Hughes K., 1999, The Accidental Diplomat. Dilemmas of the Trailing Spouse. Putnam Valley, Aletheia Publications.

Jacobs P., S. Dumont, T. Turcotte et D. Anderson, 2011, « Evaluating the Economic Loss of Caregiving for Palliative Care Patients », Journal of Palliative Care, 27 : 210-215.

KröGer T. et J. SiPILÄ (dir.), 2005, Overstretched. European Families up Against the Demands of Work and Care. Oxford, Blackwell Publishing.

LAÉ J.-F., 2003, « L'intimité : une histoire longue de la propriété de soi », Sociologie et sociétés, $35,2: 139-147$.

Le Borgne-Uguen F. et M. Rebourg (dir.), 2012, L'entraide familiale : régulations juridiques et sociales. Rennes, Presses universitaires de Rennes.

Loriol M., 2016, «Le travail diplomatique et l'intime », Socio, $7: 45-64$, consulté sur Internet (http://socio.revues.org/2382), le 25 mars 2019.

Lozerand E., 2015, «Penser les individus du monde », Socio, 5 : 139-161, consulté sur Internet (http://socio.revues.org/1933), le 25 mars 2019.

ORGANISATION DE COOPÉRATION ET DE DÉVELOPPEMENT ÉCONOMIQUE (OCDE), 2011, The Future of Families to 2030. A Synthesis Report, consulté sur Internet (https://www.oecd.org/ futures/49093502.pdf), le 2 octobre 2019.

Pailhé A. et A. Solaz (dir.), 2009, Entre famille et travail. Des arrangements de couples aux pratiques des employeurs. Paris, La Découverte.

Papadaniel Y., M.-A. Berthod et N. Brzak, 2017, « Des individus sur le fil » : 161-183, in M. Blondet et M. Lantin Mallet, Anthropologies réflexives. Lyon, Presses universitaires de Lyon. 
Perrig-Chiello P., F. HöPflinger et B. Schnegg, 2010, Pflegende Angehörige von älteren Menschen in der Schweiz. Schlussbericht : SwissAgeCare-2010 - Forschungsprojekt im Auftrag von Spitex-Schweiz, consulté sur Internet (https:/www.spitex.ch/files/CEAAGB6/ SwissAgeCare-2010---Schlussbericht), le 25 mars 2019.

Perrot M., 2009, Histoire de chambres. Paris, Seuil.

Trabut L. et F. Weber, 2009, « Comment rendre visible le travail des aidants : le cas des politiques de la dépendance en France », Idées économiques et sociales, 158, 4 : 13-22, consulté sur Internet (DOI : 10.3917/idee.158.0013), le 22 septembre 2019.

Vigarello G., 2014, Le sentiment de soi : histoire de la perception du corps (XVI $-X X^{e}$ siècle). Paris, Seuil.

Weber F., S. Gojard et A. Gramain (dir.), 2003, Charges de famille. Dépendance et parenté dans la France contemporaine. Paris, La Découverte.

\section{RÉSUMÉ - ABSTRACT-RESUMEN}

Politiques publiques et intimité face à la maladie grave : situer le rôle des proches aidants en Suisse

Ce texte traite de l'emprise que peuvent avoir — ou non - les politiques publiques sur l'engagement des proches auprès de membres de la famille gravement malades, tout particulièrement les conjoints. Lors d'une enquête anthropologique menée en Suisse romande (francophone) entre 2012 et 2016, nous avons cherché à comprendre comment ces derniers parvenaient à articuler travail, famille et accompagnement et, par conséquent, à endosser le rôle de proche aidant promu par les services de santé publique. Sur la base des trente-neuf situations d'aide informelle en fin de vie que nous avons documentées, nous montrons en nous appuyant concrètement, ici, sur cinq d'entre elles — que les individus situés en première ligne se retrouvent affectés à un espace intime où le partage est parfois difficile, voire impossible. Ce repli dans l'intimité ne participe pas nécessairement à une quelconque construction de soi ni à un renforcement positif des liens affectifs. Il amplifie au contraire les doutes et incertitudes sur l'évolution de la maladie et l'avenir des relations ; ce repli confine les proches aidants dans un espace où les questionnements ne trouvent pas de réponse. Dans cette perspective, les différences de trajectoire et de destin entre le malade et les proches se révèlent dans ce qui peut être partagé et ce qui doit être tu. La sphère intime peut dès lors s'apparenter à une zone d'inconfort susceptible de mettre ces proches en porte-à-faux avec les programmes de politiques publiques qui cherchent à mieux les soutenir et, simultanément, à les mobiliser pour répondre aux défis du vieillissement de la population et à la chronicisation des maladies.

Mots clés : Papadaniel, Berthod, intimité, maladie, fin de vie, politiques publiques, proches aidants, aidants informels, famille, Suisse 


\section{Public Policies and Intimacy Towards Severe Illness: Situating the Role of "Family Caregivers" in Switzerland}

This text explores the role public policies play-or not-in the commitment relatives show to supporting their severely ill family members, especially spouses. During an anthropological study conducted in the French-speaking part of Switzerland between 2012 and 2016, we aimed to understand how the former dealt with the interconnection of work, family and care; and, consequently, take on the role of "family caregiver" that is promoted by public health systems. Based on thirty-nine end-of-life situations that we documented, where informal care was provided, we show-referring concretely here to five of them - that the individuals on the frontline are assigned to an intimate space where sharing is challenging, at times even impossible. This withdrawal into intimacy does not contribute to any construction of the self nor to any positive reinforcement of affective bonds. On the contrary, it amplifies doubts and uncertainties regarding the development of the illness and the future of the relations; this withdrawal confines these "family caregivers" to a space where questioning finds no answers. From this point of view, the differences of trajectories and destinies between the sick person and the relatives are to be seen in the interstices of what can be shared and what has to be silenced. The intimate sphere appears then as a zone of discomfort, that can place these relatives at odds with public health programs that strive to better support them and, simultaneously, to mobilize them to face the challenges posed by an aging population and the chronicization of illnesses.

Keywords: Papadaniel, Berthod, Intimacy, Illness, End-of-life, Public Policies, Family Caregivers, Informal Caregivers, Family, Switzerland

Políticas públicas e intimidad frente a la enfermedad grave: situar el rol de los «cuidadores familiares» en Suiza

Este artículo aborda la influencia que pueden tener -o no- las políticas públicas sobre el compromiso de los familiares ante los miembros de la familia gravemente enfermos, en particular los cónyuges. Durante una encuesta antropológica realiza en Suiza romanda (francófona) entre 2012 y 2016, tratamos de comprender cómo estos últimos lograban articular trabajo, familia y acompañamiento y, consecuentemente, asumir el rol de «cuidador familiar» promovido por los dispositivos de salud pública. Sobre la base de treinta y nueve situaciones de ayuda informal durante la agonía que documentamos, mostramos -apoyándonos concretamente en cinco casos- que los individuos situados en primera línea se hayan asignados a un espacio íntimo en donde compartir es a veces difícil y muchas veces imposible. Este repliegue en la intimidad no forma parte necesariamente de alguna construcción de símismo ni a de un refuerzo positivo de las relaciones afectivas. Por lo contrario, amplifica las dudas y las incertitudes sobre la evolución de la enfermedad y el futuro de las relaciones: este repliegue los confina en un espacio en donde los cuestionamientos no encuentran respuestas. Desde esa perspectiva, las diferencias de las trayectorias y los destinos entre el enfermo y sus familiares se revelan en los intersticios entre lo que puede ser compartido y lo que se debe callar. La esfera de lo íntimo puede, así pues, semejarse a una zona de incomodidad susceptible de situar a los familiares en un saledizo con los programas de políticas públicas que buscan sostenerlos más adecuadamente y, simultáneamente, movilizarlos para responder a los retos que conlleva el envejecimiento de la población y las enfermedades crónicas.

Palabras clave: Papadaniel, Berthod, intimidad, enfermedad, fin de vida, políticas públicas, cuidadores familiares, cuidadores informales, familia, Suiza 


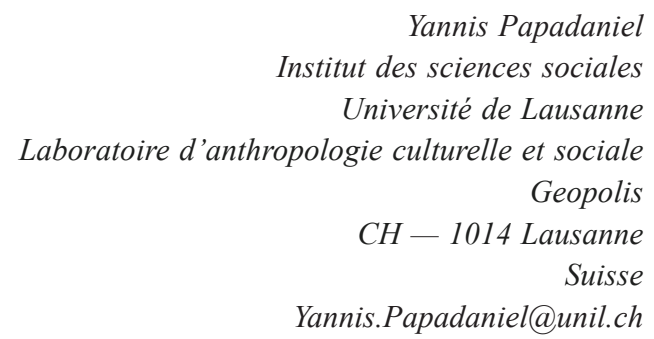

Marc-Antoine Berthod Laboratoire de recherche santé-social (LaReSS) Haute école de travail social Lausanne (Haute école spécialisée de Suisse occidentale) Chemin des Abeilles 14 $\mathrm{CH}-1010$ Lausanne Suisse Marc-Antoine.Berthod@eesp.ch 